\title{
Confronting the Gaia and NLTE spectroscopic parallaxes for the FGK stars
}

\author{
Tatyana Sitnova, Lyudmila Mashonkina and Yury Pakhomov \\ Institute of Astronomy, Russian Academy of Sciences, \\ Pyatnitskaya 48, 119017, Moscow, Russia \\ email: sitnova@inasan.ru
}

\begin{abstract}
The understanding of the chemical evolution of the Galaxy relies on the stellar chemical composition. Accurate atmospheric parameters is a prerequisite of determination of accurate chemical abundances. For late type stars with known distance, surface gravity (log g) can be calculated from well-known relation between stellar mass, $T_{\text {eff }}$, and absolute bolometric magnitude. This method weakly depends on model atmospheres, and provides reliable log g. However, accurate distances are available for limited number of stars. Another way to determine $\log \mathrm{g}$ for cool stars is based on ionisation equilibrium, i.e. consistent abundances from lines of neutral and ionised species. In this study we determine atmospheric parameters moving step-bystep from well-studied nearby dwarfs to ultra-metal poor (UMP) giants. In each sample, we select stars with the most reliable $T_{\text {eff }}$ based on photometry and the distance-based log g, and compare with spectroscopic gravity calculated taking into account deviations from local thermodinamic equilibrium (LTE). After that, we apply spectroscopic method of $\log g$ determination to other stars of the sample with unknown distances.
\end{abstract}

Keywords. stars: fundamental parameters, abundances.

Nearby dwarfs, -2.6 $\leqslant[\mathrm{Fe} / \mathrm{H}] \leqslant 0.2$. For 20 stars, with accurate non-spectroscopic atmospheric parameters, namely, $T_{\text {eff }}$ from the infrared flux method (IRFM, Alonso et al. 1996, Casagrande et al. 2011) and log g from the Hipparcos parallax (van Leeuwen2007), which was measured with an error of no more than $10 \%$ we found consistent within 0.06 dex NLTE abundances from lines of Fe I and Fe II. For other 30 stars of the sample with larger parallax errors we determined spectroscopic log g. NLTE leads to up to 0.5 dex higher $\log$ g compared to LTE. Our whole stellar sample has 22 stars in common with the Gaia DR1 (Gaia Collaboration et al. 2016). Employing the Gaia DR1 and Hipparcos parallaxes leads to consistent surface gravities for all our stars within $80 \mathrm{pc}$ from the Sun and, thus, consistent spectroscopic and distance based surface gravities. For the more distant stars, up to $\mathrm{d}=250 \mathrm{pc}$, our spectroscopic determinations were found to agree well with $\log \mathrm{g}_{\text {Gaia }}$, i.e. $\Delta \log \mathrm{g}($ Gaia - Sp) $=-0.01 \pm 0.07$, while systematically lower $\log g$ are obtained, when using the Hipparcos parallaxes, with $\Delta \log g$ (Hipparcos $\mathrm{Sp})=-0.15 \pm 0.12$ for the stars more distant than $80 \mathrm{pc}$. This proves that the NLTE spectroscopic method can provide reliable surface gravity for distant objects.

Giants, $-4.0 \leqslant[\mathrm{Fe} / \mathrm{H}] \leqslant-1.5$. Our giant sample contains 36 stars from dwarf spheroidal galaxies (dSphs) Sculptor, Ursa Minor, Sextans, Fornax, Boötes I, Ursa Major II, and Leo IV, and 24 stars from the MW halo. $T_{\text {eff }}$ were obtained with calibration from Ramírez \& Meléndez 2005 for the V-IJK colours. For giants with distance based log g and $[\mathrm{Fe} / \mathrm{H}]$ $\geqslant-3.7$ we found consistent abundances from Fe I and Fe II and Ti I and Ti II in NLTE. NLTE leads to positive abundance difference from neutral and ionised species in giants with $[\mathrm{Fe} / \mathrm{H}] \leqslant-3.7$. For MW halo stars we determined spectroscopic log g, the six stars among them have Gaia parallax measurements. For the four stars, our $\log \mathrm{g}_{s p}$ are con- 
sistent with $\log \mathrm{g}_{\text {Gaia }}$ within the error bars, and the difference log g (Sp - Gaia) does not exceed 0.11 dex. For example, for CD-38 245, $\log \mathrm{g}_{\text {Gaia }}=1.91$, and we adopted parameters 4900/2.0/-3.9, which lead to Fe I - Fe II = 0.11 dex, and Ca I - Ca II = -0.05 \pm 0.07 dex in NLTE. An outlier is HD 8724, with log g (Gaia-Sp) $=-0.76$ dex despite of small statistical, $\sigma\left(\log \mathrm{g}_{\text {Gaia }}\right)=0.08 \mathrm{dex}, \sigma\left(\log \mathrm{g}_{S p}\right)=0.24 \mathrm{dex}$, and systematic errors, $\Delta \log$ $\mathrm{g}(\mathrm{Sp}-$ Gaia $)=-0.07$ dex. To reconcile the Fe I and Fe II abundances with $\log \mathrm{g}_{\text {Gaia }}=$ $2.05, T_{\text {eff }}$ of HD 8724 should be increased by $\simeq 400 \mathrm{~K}$. This seems very unlikely, because all estimates, based on the IRFM are close to $T_{\text {eff }}=4560 \mathrm{~K}$ derived in this study.

For ultra $M P$ halo stars, $[\mathrm{Fe} / \mathrm{H}] \leqslant-4.0$ distances are not available, and reliable $\mathrm{Fe}$ II lines cannot be detected. We selected 11 UMP stars, where Ca I and Ca II lines can be detected in their observed spectra. We adopted photometric $T_{\text {eff }}$ and $\log \mathrm{g}$ from evolutionary tracks (Yi et al. 2004), and checked Ca I-II NLTE ionisation equilibrium. For all stars of the sample, Ca I and Ca II agree in NLTE within error bars. An abundance difference Ca I - Ca II does not exceed 0.16 dex in absolute value in NLTE, while it is negative, and reaches -0.90 dex in LTE. Ca I/Ca II NLTE ionization equilibrium can be used for determination of atmospheric parameters of UMP stars.

Conclusions. We found that, in wide range of luminosity and metallicity, different methods of atmospheric parameter determination based on photometry, distances, NLTE ionisation equilibrium, evolutionary tracks can provide consistent atmospheric parameters for given star. We also note that $T_{\text {eff }}$-colour calibrations are not available for $[\mathrm{Fe} / \mathrm{H}] \leqslant-4$ stars, but desired. For UMP stars, determination of $T_{\text {eff }}$ by extrapolating the calibration to lower $[\mathrm{Fe} / \mathrm{H}]$ leads to large uncertainty. From independent spectroscopic method we confirm that Gaia DR1 parallaxes are accurate.

\section{Acknowledgments}

This work has made use of data from the European Space Agency (ESA) mission Gaia (https://www.cosmos.esa.int/gaia), processed by the Gaia Data Processing and Analysis Consortium (DPAC, https://www.cosmos.esa.int/web/gaia/dpac/consortium). Funding for the DPAC has been provided by national institutions, in particular the institutions participating in the Gaia Multilateral Agreement. The authors are grateful to IAU and the grant on Leading Scientific Schools 9951.2016.2. A part of this study is carried out within a project 'The Formation and Evolution of the Galactic Halo', International Space Science Institute, Bern, Switzerland. We made use the ESO Science Archive Facility, SIMBAD, MARCS, 2MASS, SDSS, and VALD databases.

\section{References}

Alonso, A., Arribas, S., \& Martinez-Roger, C. 1996, A\&SAS, 117, 227

Casagrande, L., Schönrich, R., Asplund, M., Cassisi, S., Ramírez, I., Meléndez, J., Bensby, T., \& Feltzing, S. 2011, A\&A, 530, A138

Gaia Collaboration, Brown, A. G. A., Vallenari, A., Prusti, T., de Bruijne, J. H. J., Mignard, F. et al. 2016, A\&A, 595, A2

Ramírez, I. \& Meléndez, J. 2005, ApJ, 626, 465

van Leeuwen, F. 2007, $A \& \& A, 474,653$

Yi, S. K., Demarque, P., \& Kim, Y.-C. 2004, Ap\&SS, 291, 261 